\title{
Hungry irony
}

\author{
Nancy C. Andrews
}

Duke University School of Medicine, Durham, North Carolina, USA.

\begin{abstract}
Iron-deficient individuals experience a loss of appetite that can be restored with iron supplementation. It has been proposed that iron influences the satiety hormone leptin; however, a direct link between iron and leptin has remained elusive. In this issue of the $\mathrm{JCl}$, Gao and colleagues demonstrate an inverse relationship between adipocyte iron and leptin that is mediated by iron-dependent activation of cAMP-responsive element binding protein (CREB), the transcription factor that represses leptin transcription. Together, the results of this study provide a mechanistic connection between dietary iron and the appetite-regulating hormone leptin.
\end{abstract}

and cultured cells. In animals fed a highiron diet, both leptin mRNA and serum leptin levels were decreased. The highiron diet resulted in a two-fold increase in hepatic iron levels and more than a threefold increase in adipocyte iron. Moreover, adipocyte-specific deletion of the iron exporter ferroportin, which resulted in iron loading in adipocytes, reduced serum leptin levels. Conversely, in a murine model of hereditary hemochromatosis, adipocyte iron levels were decreased and leptin levels were increased. The same inverse correlation between adipocyte iron status and leptin mRNA expression was also observed in an adipocyte cell line.

Gao and colleagues determined that increased cellular iron causes an increase in phosphorylation of cAMP-responsive element binding protein (CREB) that is independent of the canonical CREB activators cyclic AMP and calcium. CREB phosphorylation was associated with inhibition of leptin transcription via CREB binding at sites that were detected in the putative leptin promoter (Figure 1). Moreover, expression of a dominant-negative CREB protein in cultured adipocytes rendered the leptin promoter insensitive to iron status.

\section{Remaining questions}

The work by Gao et al. raises several interesting questions. First, while mice on a high-iron diet were shown to have lower leptin levels and eat more chow than animals fed standard chow, the body weights of iron-fed mice were not increased. Additionally, measurements of fat and lean body mass in high-iron diet-fed mice were indistinguishable from animals on normal chow. Similarly, mice with increased adipocyte iron due to adipocyte-specific inactivation of ferroportin showed a leptinassociated change only in eating behavior, but not in other metrics of body size and composition. Together, these observations fit with the lack of association between ferritin and BMI described in human subjects. However, it is not clear why increased food consumption does not translate into measurable differences in body parameters. 


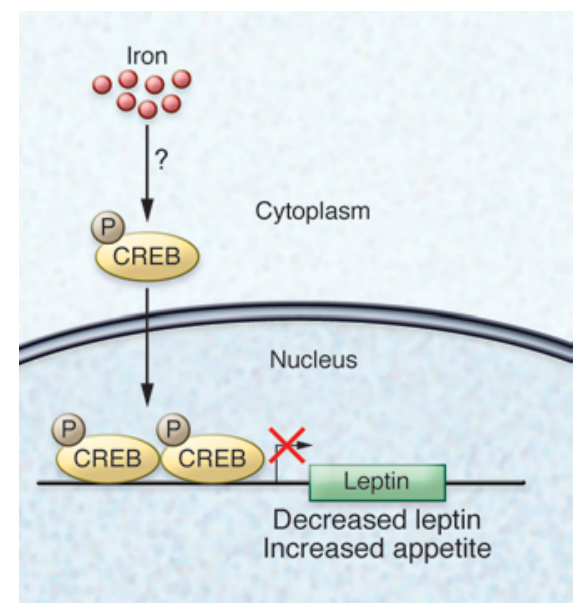

Figure 1. The transcription factor CREB connects iron status to leptin expression in adipocytes. Elevation of intracellular iron in adipocytes results in CAMP- and calcium-independent phosphorylation of CREB. Phosphorylated CREB binds 2 sites within the putative leptin promoter and inhibits transcription. Decreased leptin, in turn, increases appetite.

Second, the link between iron and CREB phosphorylation is still unknown. Others have reported that decreased intracellular iron is associated with phosphorylation or activation of CREB in a variety of cell types, possibly related to decreased reactive oxygen species (5-7), but the precise molecular details remain obscure. There is abundant evidence that cellular iron status not only influences gene expression posttranscriptionally through interactions with iron regulatory elements in noncoding regions of a limited set of mRNAs, but iron deficiency also causes increased transcription of some genes that are important in iron homeostasis (8). The mechanism for transcriptional induction in response to iron deficiency has been elusive, and CREB phosphorylation might be part of the answer. More work will need to be done to fully sort this relationship out.

Third, CREB has many targets, and it is likely that there are other downstream effects of iron-related changes in CREB activity. Iron-dependent regulation of CREB function has potential to be particularly important in cells that experience a wide physiological range in iron-storage capacity, such as intestinal epithelial cells, tissue macrophages, and hepatocytes. The broader implications of such an effect remain to be elucidated.

While there is substantial literature associating iron overload with diabetes and insulin resistance, the molecular mechanisms are incompletely understood. Clearly, the interaction between iron homeostasis and systemic energy metabolism is complex, and the report by Gao et al. contributes one additional piece to the puzzle.

\section{Acknowledgments}

N.C. Andrews is supported by NIH grant R01 DK089705.

Address correspondence to: Nancy C. Andrews, Duke University School of Med- icine, 125 Davison Building, Box 2927 Med Ctr., Durham, North Carolina 27705, USA. Phone: 919.684.2455; E-mail: nancy. andrews@duke.edu.

1. Lawless JW, Latham MC, Stephenson LS, Kinoti $\mathrm{SN}$, Pertet AM. Iron supplementation improves appetite and growth in anemic Kenyan primary school children. J Nutr. 1994;124(5):645-654.

2. Topaloglu AK, Hallioglu O, Canim A, Duzovali O, Yilgor E. Lack of association between plasma leptin levels and appetite in children with iron deficiency. Nutrition. 2001;17(7-8):657-659.

3. Isguven P, Arslanoglu I, Erol M, Yildiz M, Adal E, Erguven M. Serum levels of ghrelin, leptin, IGF-I, IGFBP-3, insulin, thyroid hormones and cortisol in prepubertal children with iron deficiency. Endocr J. 2007;54(6):985-990.

4. Gao Y, et al. Adipocyte iron regulates leptin and food intake. JClin Invest. 2015;125(9):3681-3691.

5. Zhou J, Ye S, Fujiwara T, Manolagas SC, Zhao H. Steap4 plays a critical role in osteoclastogenesis in vitro by regulating cellular iron/reactive oxygen species (ROS) levels and cAMP response element-binding protein (CREB) activation. J Biol Chem. 2013;288(42):30064-30074.

6. Zaman K, et al. Protection from oxidative stress-induced apoptosis in cortical neuronal cultures by iron chelators is associated with enhanced DNA binding of hypoxia-inducible factor- 1 and ATF-1/CREB and increased expression of glycolytic enzymes, p21(waf1/cip1), and erythropoietin. J Neurosci. 1999;19(22):9821-9830.

7. Choi EY, et al. Transcriptional regulation of IL-8 by iron chelator in human epithelial cells is independent from NF- $\kappa \mathrm{B}$ but involves ERK1/2- and p38 kinase-dependent activation of AP-1. J Cell Biochem. 2007;102(6):1442-1457.

8. Chen H, et al. Systemic regulation of Hephaestin and Ireg1 revealed in studies of genetic and nutritional iron deficiency. Blood. 2003;102(5):1893-1899. 